\title{
Can Wastewater be Used to Improve our COVID-19 Surveillance Efforts?
}

\author{
SHANNON MCGINNIS ${ }^{1}$; SHANE MCLOUGHLIN ${ }^{1}$; TIFFANY BUTURLA ${ }^{1}$; NISHITA \\ D'SOUZA2; JOSÉ LOJO ${ }^{3}$; HEATHER M MURPHY' \\ ${ }^{1}$ Department of Epidemiology and Biostatistics, College of Public Health, Temple University, \\ Philadelphia, PA, USA \\ ${ }^{2}$ The Water Quality, Environmental and Molecular Microbiology Laboratory, Michigan State University, \\ East Lansing, MI, USA \\ 3Philadelphia Department of Public Health, Philadelphia, PA, USA
}

Correspondence: shannonmarcail@gmail.com (Shannon McGinnis)

\begin{abstract}
As the spread of COVID-19 continues to significantly impact daily life in the United States and globally, there is a need for a clear understanding of disease trends in communities. Traditional methods that rely on counting individual cases often result in underreporting due to limited access to testing or healthcare. This issue is further exacerbated by the spread of COVID-19 by asymptomatic or presymptomatic individuals who may not seek testing. Historically, wastewater surveillance has been used to provide population-level data on infectious disease trends in communities. Data collected through wastewater surveillance is currently being used to advise public bealth control measures, such as vaccination campaigns, and to detect local outbreaks before cases are reported to public health authorities. For this reason, researchers around the globe are analyzing wastewater samples for SARS-CoV-2 (the virus that causes COVID-19) to assist in our response to the existing COVID-19 pandemic. This commentary discusses the potential utility of wastewater-based surveillance to advise public bealth control strategies for COVID-19 and discusses how it may be used to strengthen local surveillance efforts in Philadelphia.
\end{abstract}

\section{Introduction}

Understanding trends in COVID-19 prevalence in a community is key to guiding public health policy. Policymakers around the world have relied on COVID-19 case counts to initiate social distancing policies, to determine when to enter defined stages of reopening, and to set travel advisories. However, traditional surveillance systems that rely on predominately testing of symptomatic individuals leads to an underrepresentation of the disease prevalence. In the case of COVID-19, a lack of access to testing, long wait times, confusion about when to get tested or who should get tested, delays in receiving results, and fear of needing to quarantine in the event of a positive test have prevented or deterred some individuals from seeking testing. ${ }^{1,2}$ Further, asymptomatic individuals (which may represent $18-31 \%$ of $\operatorname{cases}^{3,4}$ ), may never seek testing and continue to spread COVID-19 in their community without contributing to these case counts. 
Wastewater-based epidemiology is a method of detecting the presence of certain pathogens in a population without relying on individual testing. Using this strategy, wastewater samples are collected from the inlets of wastewater treatment plants or outlets of individual buildings or complexes and analyzed using quantitative polymerase chain reaction methods, qPCR, (or similar strategies) to detect pathogen-specific genetic material. By doing so, wastewater surveillance allows for a small number of samples to provide data on entire communities, making it more cost-effective and less invasive than relying on testing individuals.

Wastewater epidemiology has been used to detect and prevent disease outbreaks such as polio, hepatitis $\mathrm{A}$, and norovirus, 5,6 around the world and for this reason, is a growing area of research in the context of the current COVID-19 pandemic. This commentary examines the strengths and weaknesses of wastewater-based surveillance for SARS-CoV-2 (the virus that causes COVID-19) and discusses how it may be used to strengthen local surveillance efforts in Philadelphia.

\section{History of Wastewater Epidemiology}

Wastewater-based epidemiology has been used since the 1980s to find associations between pathogen concentrations in wastewater and morbidity in communities. For example, in 1984, sewage monitoring was used to track the progression of a poliovirus outbreak in Finland and to assess the success of a vaccination campaign. ${ }^{7}$ In 1988, sewage monitoring was again used to track a polio outbreak in Israel when sewage isolates in northern communities were genetically linked to samples isolated from southern communities, allowing for a better understanding of how and when the disease was spread. ${ }^{7}$ Similarly in Egypt, sewage surveillance for poliovirus has been ongoing since the early 2000s and has successfully detected wild-type poliovirus in samples collected in 2008 and again in 2010, which were found to be genetically linked to poliovirus strains isolated in in South Asia. ${ }^{6}$ While these incidents did not result in cases of poliomyelitis in Egypt, they demonstrate the ability of wastewater monitoring to detect infectious disease cases that would have otherwise gone unnoticed in the community. ${ }^{6}$

In addition to improving community disease surveillance and enhancing epidemiologic understanding of local outbreaks, the detection of poliovirus in wastewater has also been used to target communities for vaccination campaigns. For example, in Israel, an outbreak of polio in 2013-2014, which was identified through wastewater surveillance, quickly triggered a widespread vaccination campaign. ${ }^{8}$ Evaluation of this vaccination campaign concluded that it was successful in slowing transmission of the disease and preventing the outbreak from reaching other areas. ${ }^{8}$ While there is currently no vaccine approved for COVID-19, in future years a similar strategy may be used to target areas experiencing local COVID-19 epidemics.

In addition to poliovirus, wastewater surveillance may also improve community surveillance of other pathogens such as norovirus and Hepatitis A. ${ }^{9}$ For example, during a norovirus outbreak in Sweden, wastewater monitoring detected norovirus in wastewater samples 2-3 weeks before the first community cases were identified. ${ }^{9}$ Similarly, monitoring of sewage samples for Hepatitis A in Brazil and Italy allowed for better understanding of the epidemiology of Hepatitis A in these communities by highlighting geographical differences and seasonal realtionships. ${ }^{10,11}$ By allowing for early detection of local outbreaks as well as providing valuable epidemiologic data, wastewater-based epidemiology has proven to be a useful tool in understanding and responding to outbreaks globally. 


\section{Application to SARS-Co-2}

SARS-CoV-2 is shed in the feces of symptomatic, pre-symptomatic, and asymptomatic individuals, including some individuals who have tested negative through nasopharyngeal swab samples. ${ }^{12,13}$ SARSCoV-2 can survive in stool samples for 17-31 days, which is longer, on average, than in respiratory or serum samples (13-29 days in respiratory samples, and 11-21 days in serum samples) ${ }^{14}$. In addition, diarrhea has been reported as a symptom of COVID-19 infection in approximately $2-50 \%$ of cases, possibly occurring earlier and lasting longer than the more traditional respiratory symptoms. ${ }^{15}$

Currently, teams around the world are monitoring wastewater for SARS-CoV-2 to determine if wastewater samples may be used to guide future intervention strategies. Early examples of SARS-CoV-2 detection in sewage samples occurred in the Netherlands, ${ }^{16}$ Australia, ${ }^{17}$ and France $^{18}$ and today wastewater monitoring is ongoing in Canada, Europe, South America, Asia, and Australia. ${ }^{19}$ In the United States, the Centers for Disease Control and Prevention (CDC) and the U.S. Department of Health and Human Services (HHS) initiated a Wastewater Surveillance System (NWSS) in response to the COVID19 pandemic. ${ }^{20}$ The goal of this system is to synthesize wastewater surveillance data collected across the United States in order to maximize the impact of these projects and improve comparability across communities. ${ }^{20}$ Some of these global projects have successfully detected concentrations of SARS-CoV-2 in wastewater prior to local disease outbreaks ${ }^{21,22}$ and others have found associations between SARS$\mathrm{CoV}-2$ concentrations in wastewater and the number of COVID-19 cases reported locally.23,24

In addition to widespread community monitoring, wastewater samples have also been collected from individual buildings to identify a potential COVID-19 outbreak before it spreads. At the University of Arizona, SARS-CoV-2 was detected in a wastewater sample collected outside of a dorm building. ${ }^{25}$ This prompted a building-wide testing and isolation strategy which likely prevented a widespread outbreak on the campus. ${ }^{25}$ Other colleges and universities have since taken steps to investigate this approach to controlling outbreaks on their own campuses (some of which are included in this online map: https://ucmerced.maps.arcgis.com/apps/opsdashboard/index.html\#/c778145ea5bb4daeb58d31afee3 $\underline{89082})^{26-28}$

\section{Potential for Fecal-Oral Transmission}

High concentrations of SARS-CoV-2 in sewage may raise concerns about the potential for fecal-oral transmission. In fact, there was a documented outbreak of the closely related SARS-CoV-1 (SARS) that occurred in an apartment building in Hong Kong, when aerosolized urine or fecal matter (through faulty plumbing systems or by toilet flushing) resulted in 321 illnesses and 42 deaths. ${ }^{29}$ Despite this, current evidence has determined that the persistence of SARS-CoV-2 in wastewater does not seem to be significant due to its low survivability in water and its sensitivity to common disinfectants. ${ }^{30,31}$ However, despite its low survivability in water, viral RNA often remains intact and therefore can be used to quantify the amount of SARS-CoV-2 within wastewater even when the virus is not viable. While the presence of SARS-CoV-2 genetic material can be detected in fecal samples and untreated wastewater, at the time of publication of this article, no known fecal-oral transmission of COVID-19 has been documented. ${ }^{28}$ 


\section{Wastewater Monitoring in Philadelphia}

As a part of the global effort to detect and monitor SARS-CoV-2 concentrations in community wastewater samples, the Water, Health, and Applied Microbiology (WHAM) lab within Temple University's College of Public Health has been working with Michigan State University (MSU), the Philadelphia Water Department (PWD), and the Philadelphia Department of Public Health (PDPH) to collect this data in Philadelphia.

Since May 2020, weekly 24-hour composite wastewater samples have been collected from three water treatment plant influents (wastewater that enters the plant to undergo treatment) in Philadelphia. These three treatment plants serve the entirety of Philadelphia's wastewater treatment demands. 24-hour composite samples are collected by an autosampler which is cooled to $4^{\circ} \mathrm{C}$ at each treatment plant. A volume pre-defined by each plant is taken from the plant's influent at 30-minute intervals over the 24hour period to create the composite sample. A composite sample is meant to provide a more accurate picture of the city's wastewater characteristics than by pulling a grab sample at one point in the day, which is subject to greater variability due to changes in water use patterns throughout the day. These samples are individually processed at Temple's WHAM lab and analyzed at the Rose Lab at MSU using digital PCR (or ddPCR) procedures which involve the quantification of three SARS-CoV-2 genes (N1, N2, E). In the future, concentrations of SARS-CoV-2 in these samples will be compared to community surveillance data to determine whether wastewater data may provide insight into the trends of COVID19 in Philadelphia. Currently, the PDPH and PWD are working to determine the relationship between the number of gene copies of SARS-CoV-2 present in wastewater samples and COVID-19 trends in the communities served by each of the wastewater treatment plants. This is made more difficult by the aforementioned issues of unreported illness and asymptomatic cases. Once this relationship becomes more clear, data on the concentrations of SARS-CoV-2 in Philadelphia's wastewater may be used to predict future increases in measured case counts in the city, allowing city agencies to mobilize prevention and control efforts.

\section{Strengths and Weaknesses}

Using wastewater-based epidemiology for the detection of SARS-CoV-2 in the community is easier, more cost-effective, non-invasive, and relies on a smaller number of samples than relying on locally reported case counts. ${ }^{30}$ Unlike traditional disease surveillance, which requires individuals to seek out healthcare or testing, a single sample of untreated wastewater can offer a snapshot of population-level data, quickly providing information about an entire community. In addition, wastewater can detect SARS-CoV-2 shed by presymptomatic and asymptomatic individuals who may not seek testing or by people who do not have access to testing or healthcare, which may improve our knowledge of disease trends in the community. Wastewater may also be used to track the spread of specific emerging strains, which can assist in our understanding of their movement globally. ${ }^{29}$ Additionally, wastewater may be able to detect changes in disease prevalence in a community before traditional surveillance methods that rely on individual case counts, as viral shedding in feces can occur before other respiratory symptoms.

Despite the increasing popularity of wastewater-based epidemiology for understanding the epidemiology of COVID-19 around the world, there are limitations to this monitoring strategy. First, there is significant variability in the amount of virus shed in the feces of individuals as well as in the way sewage is collected and handled in communities (i.e., it may be mixed with stormwater, industrial wastewater etc.). This makes it difficult to define a concentration threshold in wastewater samples that corresponds with thresholds that are set using traditional surveillance. Further, wastewater monitoring from the inlet of a 
single wastewater treatment plant may represent disease cases from other surrounding communities, depending on the catchment area of each system. For this reason, it may be difficult to use wastewater to make decisions regarding a single community. For example, in Philadelphia, one of its three wastewater treatment plants serves locations in Montgomery County and Springfield Township. This means that the data provided from the sample at this site covers an area beyond the boundaries of Philadelphia, which introduces an additional variable, requiring an analysis of SARS-CoV-2 infection trends in additional communities.

In addition, there is a lack of research on how environmental conditions such as $\mathrm{pH}$, sunlight, temperature, etc. may impact the persistence and detection of SARS-CoV-2 in wastewater, thereby impacting the interpretation of data collected during different seasons or in different locations. Finally, there is a lack of a consensus around the best methods for concentrating wastewater samples for qPCR analysis, causing limited comparability between studies conducted in different areas by various research teams. ${ }^{30,32}$

\section{Conclusions}

Despite these limitations, ongoing surveillance of SARS-CoV-2 in community wastewater systems may complement traditional surveillance methods, especially in areas where testing is limited, to guide resource allocation and social distancing policies. In future years, wastewater may serve as an early warning indicator of a re-emergence of disease cases, as has been done around the world for poliovirus. ${ }^{5,8}$ For this reason, ongoing collection and analysis of wastewater samples for SARS-CoV-2 as well as research that compares various sampling, processing and detection procedures for wastewater samples may become critical for our response to the current pandemic and for our ongoing efforts to prevent or respond to future outbreaks.

\section{Disclosures and Conflicts of Interest}

The authors report no competing interests.

\section{Funding Sources}

The Philadelphia Department of Public Health provided funding for the wastewater surveillance project described in this manuscript. No funding was provided for the development of this commentary.

\section{Acknowledgments}

The authors thank Caroline Johnson from Philadelphia Department of Health, Joan Rose from Michigan State University, and Gary A. Burlingame from Philadelphia Water Department, for their insights and review of this article. The authors also thank the Philadelphia Water Department's Bureau of Laboratory Services for supporting wastewater sample collection. The authors acknowledge their collaborators at The Water Quality, Environmental, and Molecular Biology Laboratory at Michigan State University, specifically Matthew Flood, for performing PCR analyses. 


\section{References}

1. Haire B, Williams J. Why some people don't want to take a COVID-19 test. The Conversation. http://theconversation.com/why-some-people-dont-want-to-take-a-covid-19-test-141794.

Published July 3, 2020. Accessed October 5, 2020.

2. Souch JM, Cossman JS. A Commentary on Rural-Urban Disparities in COVID-19 Testing Rates per 100,000 and Risk Factors. J Rural Health. 2020;n/a(n/a). doi:10.1111/jrh.12450

3. Mizumoto K, Kagaya K, Zarebski A, Chowell G. Estimating the asymptomatic proportion of coronavirus disease 2019 (COVID-19) cases on board the Diamond Princess cruise ship, Yokohama, Japan, 2020. Eurosurveillance. 2020;25(10). doi:10.2807/1560-7917.ES.2020.25.10.2000180

4. Nishiura H, Kobayashi T, Miyama T, et al. Estimation of the asymptomatic ratio of novel coronavirus infections (COVID-19). Int J Infect Dis. 2020;94:154-155. doi:10.1016/j.ijid.2020.03.020

5. Berchenko Y, Manor Y, Freedman LS, et al. Estimation of polio infection prevalence from environmental surveillance data. Sci Transl Med. 2017;9(383). doi:10.1126/scitranslmed.aaf6786

6. Blomqvist S, Bassioni LE, Nasr EMEM, et al. Detection of Imported Wild Polioviruses and of Vaccine-Derived Polioviruses by Environmental Surveillance in Egypt. Appl Environ Microbiol. 2012;78(15):5406-5409. doi:10.1128/AEM.00491-12

7. Pöyry T, Stenvik M, Hovi T. Viruses in sewage waters during and after a poliomyelitis outbreak and subsequent nationwide oral poliovirus vaccination campaign in Finland. Appl Environ Microbiol. 1988;54(2):371-374. doi:10.1128/AEM.54.2.371-374.1988

8. Brouwer AF, Eisenberg JNS, Pomeroy CD, et al. Epidemiology of the silent polio outbreak in Rahat, Israel, based on modeling of environmental surveillance data. Proc Natl Acad Sci. 2018;115(45):E10625-E10633. doi:10.1073/pnas.1808798115

9. Hellmér M, Paxéus N, Magnius L, et al. Detection of Pathogenic Viruses in Sewage Provided Early Warnings of Hepatitis A Virus and Norovirus Outbreaks. Appl Environ Microbiol. 2014;80(21):6771-6781. doi:10.1128/AEM.01981-14

10. Prado T, Fumian TM, Miagostovich MP, Gaspar AMC. Monitoring the hepatitis A virus in urban wastewater from Rio de Janeiro, Brazil. Trans R Soc Trop Med Hyg. 2012;106(2):104-109. doi:10.1016/j.trstmh.2011.10.005

11. La Rosa G, Della Libera S, Iaconelli M, et al. Surveillance of hepatitis A virus in urban sewages and comparison with cases notified in the course of an outbreak, Italy 2013. BMC Infect Dis. 2014;14(1):419. doi:10.1186/1471-2334-14-419

12. Jiang X, Luo M, Zou Z, Wang X, Chen C, Qiu J. Asymptomatic SARS-CoV-2 infected case with viral detection positive in stool but negative in nasopharyngeal samples lasts for 42 days. J Med Virol. 2020;92(10):1807-1809. doi:10.1002/jmv.25941

13. Tang A, Tong Z-D, Wang H-L, et al. Detection of Novel Coronavirus by RT-PCR in Stool Specimen from Asymptomatic Child, China. Emerg Infect Dis. 2020;26(6):1337-1339. doi:10.3201/eid2606.200301

14. Zheng S, Fan J, Yu F, et al. Viral load dynamics and disease severity in patients infected with SARSCoV-2 in Zhejiang province, China, January-March 2020: retrospective cohort study. BMJ. 2020;369. doi:10.1136/bmj.m1443

15. D'Amico F, Baumgart DC, Danese S, Peyrin-Biroulet L. Diarrhea During COVID-19 Infection: Pathogenesis, Epidemiology, Prevention, and Management. Clin Gastroenterol Hepatol Off Clin Pract J Am Gastroenterol Assoc. 2020;18(8):1663-1672. doi:10.1016/j.cgh.2020.04.001

16. Medema G, Heijnen L, Elsinga G, Italiaander R, Brouwer A. Presence of SARS-Coronavirus-2 in sewage. medRxiv. July 2020:2020.03.29.20045880. doi:10.1101/2020.03.29.20045880

17. Ahmed W, Angel N, Edson J, et al. First confirmed detection of SARS-CoV-2 in untreated wastewater in Australia: A proof of concept for the wastewater surveillance of COVID-19 in the community. Sci Total Environ. 2020;728:138764. doi:10.1016/j.scitotenv.2020.138764 
18. Wurtzer S, Marechai V, Mouchei J, et al. Time course quantitative detection of SARS-CoV-2 in Parisian wastewaters correlates with COVID-19 confirmed cases. MedRxiv. 2020.

19. Status of environmental surveillance for SARS-CoV-2 virus. https://www.who.int/news$\mathrm{room} / \mathrm{commentaries/detail/status-of-environmental-surveillance-for-sars-cov-2-virus.} \mathrm{Accessed}$ December 4, 2020.

20. CDC. National Wastewater Surveillance System. Centers for Disease Control and Prevention. https://www.cdc.gov/coronavirus/2019-ncov/cases-updates/wastewater-surveillance.html. Published October 23, 2020. Accessed December 4, 2020.

21. Wurtzer S, Marechal V, Mouchel J-M, et al. Evaluation of Lockdown Impact on SARS-CoV-2 Dynamics through Viral Genome Quantification in Paris Wastewaters. Epidemiology; 2020. doi:10.1101/2020.04.12.20062679

22. Randazzo W, Truchado P, Cuevas-Ferrando E, Simón P, Allende A, Sánchez G. SARS-CoV-2 RNA in wastewater anticipated COVID-19 occurrence in a low prevalence area. Water Res. 2020;181:115942. doi:10.1016/j.watres.2020.115942

23. Nemudryi A, Nemudraia A, Wiegand T, et al. Temporal Detection and Phylogenetic Assessment of SARS-CoV-2 in Municipal Wastewater. Cell Rep Med. 2020;1(6):100098. doi:10.1016/j.xcrm.2020.100098

24. Peccia J, Zulli A, Brackney DE, et al. SARS-CoV-2 RNA concentrations in primary municipal sewage sludge as a leading indicator of COVID-19 outbreak dynamics. medRxiv. June 2020:2020.05.19.20105999. doi:10.1101/2020.05.19.20105999

25. Peiser J. The University of Arizona says it caught a dorm's covid-19 outbreak before it started. Its secret weapon: Poop. Washington Post.

https://www.washingtonpost.com/nation/2020/08/28/arizona-coronavirus-wastewater-testing/. Published August 28, 2020. Accessed October 5, 2020.

26. Wastewater at $\mathrm{U}$ of Guelph residences being tested for signs of COVID-19. Guelph Mercury Tribune. https://www.guelphmercury.com/news-story/10213679-wastewater-at-u-of-guelphresidences-being-tested-for-signs-of-covid-19/. Published October 1, 2020.

27. Richtel M. Looking to Reopen, Colleges Become Labs for Coronavirus Tests and Tracking Apps. The New York Times. https://www.nytimes.com/2020/08/30/us/colleges-coronavirusresearch.html. Published September 9, 2020. Accessed October 7, 2020.

28. Walke HT, Honein MA, Redfield RR. Preventing and Responding to COVID-19 on College Campuses. JAMA. September 2020. doi:10.1001/jama.2020.20027

29. McKinney KR, Gong YY, Lewis TG. Environmental transmission of SARS at Amoy Gardens. J Environ Health. 2006;68(9):26-30; quiz 51-52.

30. Larsen DA, Wigginton KR. Tracking COVID-19 with wastewater. Nat Biotechnol. 2020;38(10):1151-1153. doi:10.1038/s41587-020-0690-1

31. Bivins A, Greaves J, Fischer R, et al. Persistence of SARS-CoV-2 in Water and Wastewater. Environ Sci Technol Lett. October 2020. doi:10.1021/acs.estlett.0c00730

32. Kitajima M, Ahmed W, Bibby K, et al. SARS-CoV-2 in wastewater: State of the knowledge and research needs. Sci Total Environ. 2020;739:139076. doi:10.1016/j.scitotenv.2020.139076

\section{Statement of Contributions}

Tiffany Burtula and Shane McLoughlin conducted a review of the literature for the development of this manuscript. All authors contributed to drafting and editing. 